Magnetic-field-induced lateral displacements of current filaments in n-GaAs

This article has been downloaded from IOPscience. Please scroll down to see the full text article.

1994 Semicond. Sci. Technol. 9373

(http://iopscience.iop.org/0268-1242/9/4/007)

View the table of contents for this issue, or go to the journal homepage for more

Download details:

IP Address: 132.199.32.109

The article was downloaded on 04/10/2010 at 12:04

Please note that terms and conditions apply. 


\title{
Magnetic-field-induced lateral displacements of current filaments in $\mathrm{n}$-GaAs
}

\author{
J Spangler, B Finger, C Wimmer, W Eberle and W Prettl \\ Institut für Angewandte Physik, Universitāt Regensburg, 93040 Regensburg, \\ Germany
}

Received 22 November 1993, accepted for publication 27 January 1994

\begin{abstract}
The formation of current filaments in n-type GaAs due to the low temperature impurity breakdown has been investigated in crossed electric and magnetic fields with a laser scanning microscope. In a highly compensated, low mobility, epitaxial layer the lateral displacement and stretching of a filament due to the Lorentz force has been observed at currents slightly larger than those required to form a filament between point contacts. The transverse elongation of the filament increases linearly with rising magnetic field strength until the filament is disrupted. In this case periodic current oscillations are generated by a repetitive filament ignition and extinction. The elongation decreases with rising current and finally vanishes even at high magnetic fields. This stiffening of a current filament is attributed to an increased electron temperature and mobility in the filament, which cause the charges of the Hall field to be accumulated at the filament borders rather than at the edges of the sample.
\end{abstract}

\section{Introduction}

High-purity semiconductors represent nonlinear dynamical systems at low temperatures, where in thermal equilibrium most of the carriers are bound to shallow impurities. The dominant nonlinearity is the autocatalytic generation of free carriers by impact ionization of shallow impurities in an external electric field. At a critical threshold field strength the system is driven into a state far from thermal equilibrium, which is accomplished by the formation of dissipative structures in the form of current filaments. At the threshold of this low temperature impurity breakdown the current increases by orders of magnitude at an almost constant electric field because of the lateral growth of a current filament. Such highly nonlinear current-voltage characteristics have been observed in practically all homogeneously doped materials and recently also with partially ordered doping structures in the form of $\delta$-layers [1].

The shape of filaments and spatial patterns of current flow in the post-breakdown regime have been reconstructed in thin p-Ge slabs and n-GaAs epitaxial layers by applying electron microscope $[2,3]$ and laser scanning [4] techniques.

The transition from the high-ohmic phase to filamentary current flow is in many cases accompanied by self-generated nonlinear oscillations under DC bias [5-11]. Investigations of thin $\mathrm{n}-\mathrm{GaAs}$ epitaxial layers have shown that small external magnetic fields may drastically affect the stability of a filament and the temporal characteristics of the oscillations [11-14]. In particular, chaotic fluctuations may be induced by very small magnetic fields oriented normally to the plane of the semiconductor layer [13]. Lorentz-forceinduced lateral movements of current filaments formed between homogeneous stripe contacts in $\mathrm{p}-\mathrm{Ge}$ have been concluded from potential probe measurements [15]. The transverse motion of a filament in crossed electric and magnetic fields has been described in terms of a semiclassical nonlinear semiconductor transport model yielding a variety of spatio-temporal dynamical phenomena [16]. However, no theory yet exists that reproduces systematically the observed current-voltage characteristics. A simple phenomenological approach has shown that the formation of a stable filament needs, in addition to drift and diffusion, an inward directed force at the filament boundaries, which was attributed to the self-attraction of the filamentary current flow [13].

In the present paper we report on reconstructions of current filaments in a rather highly compensated and low mobility $\mathrm{n}-\mathrm{GaAs}$ epitaxial layer with a laser scanning microscope. A lateral excursion of the filament due to a magnetic field normal to the plane of the sample is visualized for the first time. At low currents the filament fixed between two point contacts is stretched like a string in a constant potential gradient. At a critical magnetic field strength the filament breaks and the current flow is disrupted. This causes regular relaxation oscillations due to a repetitive ignition and extinction 
of the filamentary current flow. The lateral elongation of the filament decreases with rising current and finally vanishes. At high currents the magnetic field does not affect the coarse spatial current distribution. Only the well established instabilities at the filament border may occur, which cause multi-frequency oscillations and chaotic fluctuations [11, 17].

\section{Experimental procedure}

\subsection{Sample and current-voltage characteristics}

The sample investigated here is a $29 \mu \mathrm{m}$ thick epitaxial layer grown on a rectangular semi-insulating substrate by liquid phase epitaxy. The effective donor concentration, the compensation ratio and the $77 \mathrm{~K}$ mobility were $N_{\mathrm{D}}-N_{\mathrm{A}}=9.7 \times 10^{13} \mathrm{~cm}^{-3}, N_{\mathrm{A}} / N_{\mathrm{D}}=0.9$ and $\mu=45 \times 10^{3} \mathrm{~cm}^{2} \mathrm{~V}^{-1} \mathrm{~s}^{-1}$, respectively. Ohmic point contacts were alloyed onto opposite edges of the sample. The distance between the contacts was $3 \mathrm{~mm}$. The sample was fixed in an optical immersion cryostat in the centre of a superconducting magnet and cooled to $1.8 \mathrm{~K}$. The magnetic field was applied normal to the plane of the epitaxial layer.

The sample was biased in series with a load resistor of $100 \mathrm{k} \Omega$ by a high precision adjustable constant-voltage source. The sample exhibits the well known highly nonlinear current-voltage characteristic and shows self-sustained oscillations at the threshold of impact ionization breakdown. The current-voltage characteristic is displayed for various magnetic fields in figure 1. The hatched areas indicate the extent of oscillations in the current-voltage $(I-V)$ plane. In these areas of the $I-V$ plane virtually no definite currentvoltage relation exists. With rising average current three distinct regimes in the current-voltage characteristic may be distinguished: the pre-breakdown regime of high resistance, a regime of switching between low- and highconducting states yielding regular relaxation oscillations, and a post-breakdown regime of filamentary current flow.

The current-voltage characteristics show hysteresis, in particular the oscillatory regimes are different for

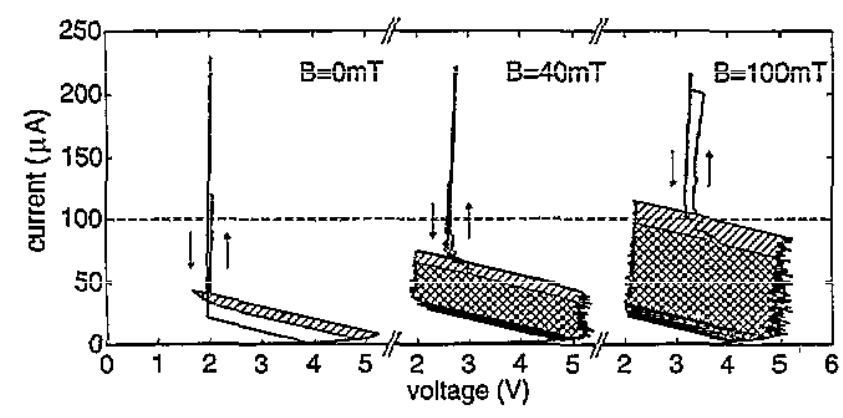

Figure 1. Current-voltage characteristics of an n-GaAs epitaxial layer for various magnetic field strengths at $T=1.8 \mathrm{~K}$. Hatched regions show the extent of selfsustained oscillation. Arrows, for stable current flow, and right and left inclined hatching in the oscillatory regimes indicate increasing and decreasing currents, respectively. increasing and decreasing current. This is indicated in figure 1 by arrows and by right and left inclined hatching, respectively. The extent of regular oscillations on the current scale increases with rising magnetic field. Thus a stable filamentary current flow at zero magnetic field is destabilized by applying a magnetic field yielding regular osciliations. At a constant bias current of, for example, $100 \mu \mathrm{A}$ the limit of stability is approached at a magnetic field of about $B=100 \mathrm{mT}$, as shown by the broken line in figure 1 . This behaviour of the present sample is unlike other materials, which have a lower compensation with mobilities of about $1 \hat{0}^{5} \mathrm{~cm}^{2} \widehat{\mathrm{V}}^{-1} \mathrm{~s}^{-1}$ or higher. In high mobility samples the regime of regular oscillations shrinks if a magnetic field is applied normal to the epitaxial layer and the constant current flow is destabilized, yielding quasiperiodic and chaotic osciliations $[1 \overline{1}, 1 \overline{4}]$. In the present sample complex oscillations could also be detected for field strengths larger than about $B=20 \mathrm{mT}$. However, the amplitudes are much smaller than those of the regular oscillations and they cannot be recognized on the scale of figure 1 .

\subsection{Scanning laser microscope}

The spatial structure of the current flow in the regime of stable filamentary current flow has been analysed by a scanning laser microscope [4]. The spatially filtered beam of a pulsed GaAlAs semiconductor laser with a wavelength of $0.8 \mu \mathrm{m}$ is scanned line by line across the sample surface. The irradiation-induced current response to the $25 \mathrm{~ns}$ long laser pulses is synchronously recorded as a function of the focal position. Scanning is achieved by a rotating drum carrying 16 individually adjustable mirrors. Subsequent lines are addressed by tilting the axis of the drum. The drum may be rotated either continuously or by a stepping drive which allows the irradiation of any point on the surface for as long as required. The light beam is focused on the epitaxial layer by a lens of $30 \mathrm{~mm}$ focal placed length inside the cryostat, giving a spot diameter of about $15 \mu \mathrm{m}$, which determines the spatial resolution of the device. The intensity of the laser beam could be varied by attenuators. The quantum energy of the laser radiation is larger than the energy gap of GaAs, hence electron-hole pairs are generated at the irradiated spot. The photosignal is recorded using a transient digitizer and the signal pattern in space is determined numerically.

The physical mechanism of this method of reconstructing the spatial extent of a current filament may be understood from the delicate balance of inward and outward directed forces on the electrons which form the filament in a state far from equilibrium. Structure-forming forces on the current flow in a semiconductor are the internal pressure of the hot electron gas, lateral diffusion, drift by local electric fields and the self-attraction of a current. In particular the filament borders, representing a phase boundary between non-equilibrium phases, are very sensitive to external perturbations. Slight variations of the electron population or localized imperfections in the material may lead to large changes in the overall 
current flow, as has been observed by far-infrared irradiation [18] or in magnetoconductivity [19] studies.

In the present case of local interband irradiation, a photoconductive signal is detected due to interband excitations. This signal is independent of the position of the irradiated spot on the sample and produces a spatially constant background photoconductivity. Extrinsic photoconductivity does not occur, owing to local charge neutrality. Ionization of shallow donors generates mobile electrons but the ionized donors stay fixed in space. Thus this process does not lead to a photoconductive signal apart from a displacement current caused by irradiation transients. Next, the interband excitation locally increases the density of free carriers and, in turn, raises the impact ionization rate of shallow donors. Above a threshold intensity the free carrier concentration may be just sufficient to trigger an impact-ionization-induced current avalanche, which is recorded as a photosignal above the level of the interband photoconductivity background. For intensities slightly above the threshold, avalanches occur at the filament borders in the transition zone between the highconducting and low-conducting phases. Because of charge neutrality these avalanches must go through the sample from the injecting contact to the drain electrode, yielding a lateral excursion of the irradiated filament border. Thus steady irradiation of a filament border causes a fluctuation of the current [17]. At higher light intensities current avalanches may also be triggered outside the filament in the regions of predominant ground state population and to a lesser degree in the filament because there the electron concentration approaches saturation. The intensities applied in the present measurements remained below this level. Thus, scanning the laser focus along a line normal to the current flow generates structures on top of the constant interband photoconductivity background which indicate the location of the filament boundaries.

\subsection{Reconstruction of the current flament}

The spatial extent of the current flow has been investigated for various magnetic field strengths $B$ as a function of the current in the post-breakdown regime. Reconstructions at $B=0$ are shown in figure 2. The top diagram displays the sample geometry with respect to the coordinate axes used in the following representations. The square and the dots correspond to the sample surface and the alloyed contacts of about $0.5 \mathrm{~mm}$ diameter. Contacts 1 and 2 were used for the present measurements. The area surrounded by broken lines has been scanned by the laser focus. The middle diagram shows a reconstruction for a current $I=$ $100 \mu \mathrm{A}$. The signal heights represent the photocurrent in excess of the interband signal detected as a function of the focus position. In the bottom diagram a signal along a line perpendicular to the centre between the contacts is plotted as function of the current. With increasing current two ridges show up which are parallel to the current flow and are symmetrically located
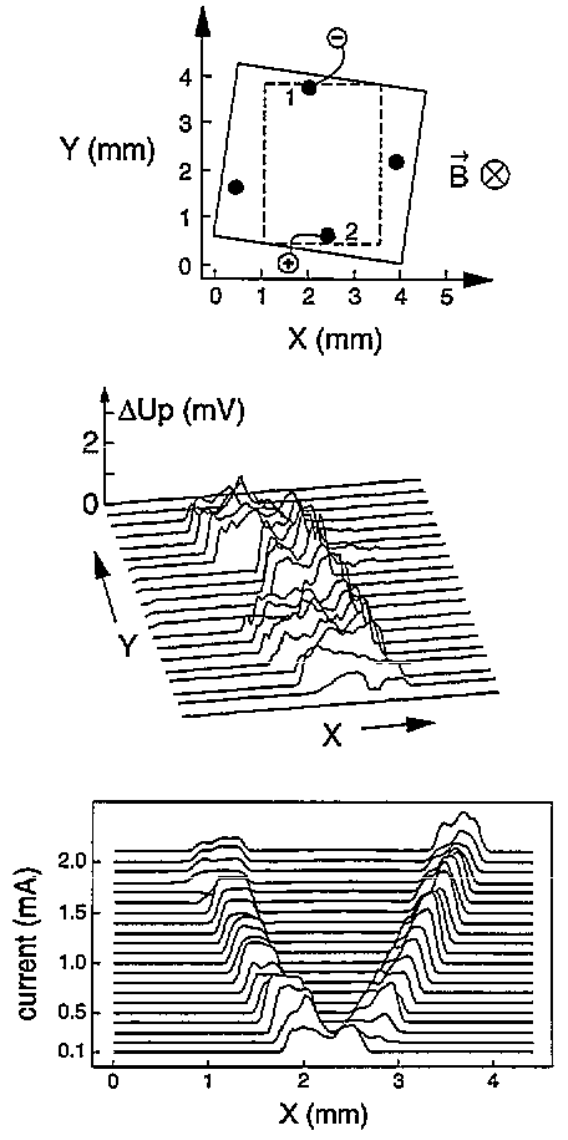

Figure 2. Top: plan view and geometry of the sample. Dots indicate point contacts. The frame surrounded by the broken line was scanned by the focused laser beam parallel to the $x$-axis. Middle: reconstruction of a filament at $I=100 \mu \mathrm{A}$ and $B=0$. The signal heights represent spatially resolved line scans of the voltage drop across the sample detected as a function of the position of the laser focus. Bottom: photosignal along a single line in the centre of the filament at $B=0$ for various bias currents.

about a line connecting the electrical contacts. These two ridges indicate the borders of the filament. The measurement shows that the increase in the current is due to a continuous lateral growth of the filament. For small currents the two ridges overlap, limiting the spatial resolution of this optical method of filament reconstruction.

Figure 3 shows the effect of a magnetic field on the filament at $100 \mu \mathrm{A}$ presented in figure 2 . Reconstructions at $B=40 \mathrm{mT}$ are displayed (top) for both orientations of the magnetic field vector and both directions of the current. The bending of the filament due to the Lorentz force is clearly observed. The situation is similar to the case of a current-carrying elastic wire fixed at the electrical contacts. The inversion of the current and the magnetic field, indicated by double arrows in the figure, yields identical structures as expected from the symmetry of the Lorentz force. In the bottom diagram the response along a single line in the centre between the contacts is plotted for various magnetic fields up to $\pm 40 \mathrm{mT}$. This recording shows the displacement of the centre of the filament as a function of the magnetic field strength. The measurements were carried at $100 \mu \mathrm{A}$ along the broken line in the 

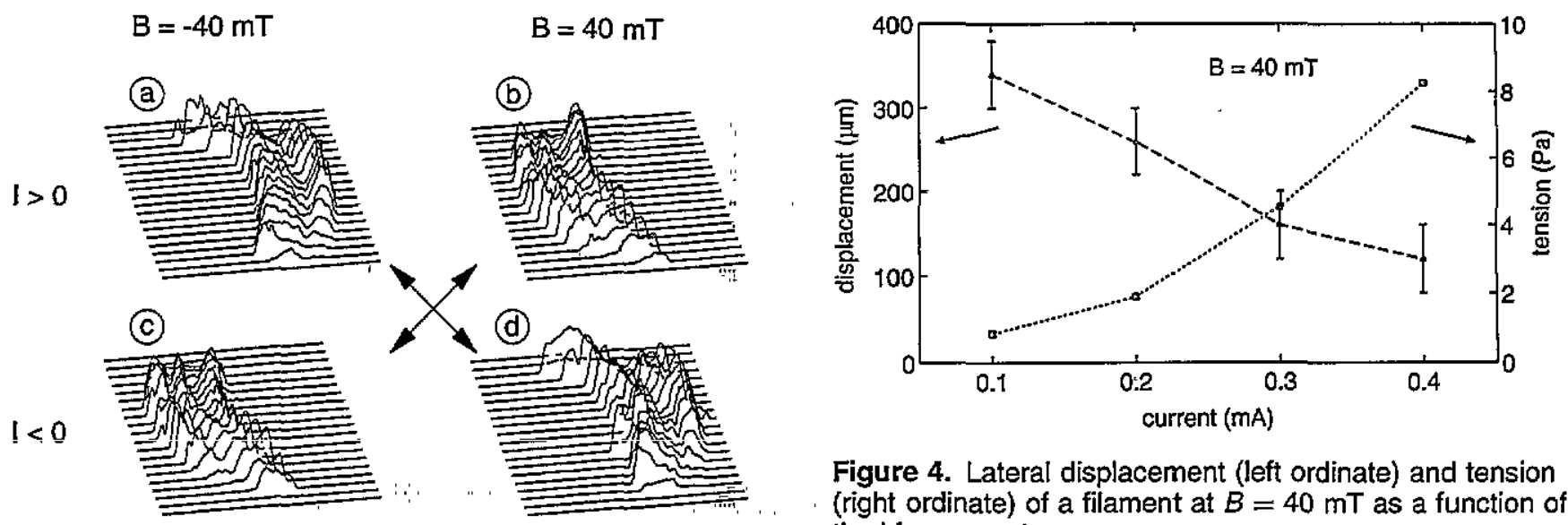

Figure 4. Lateral displacement (left ordinate) and tension (right ordinate) of a filament at $B=40 \mathrm{mT}$ as a function of the bias current.
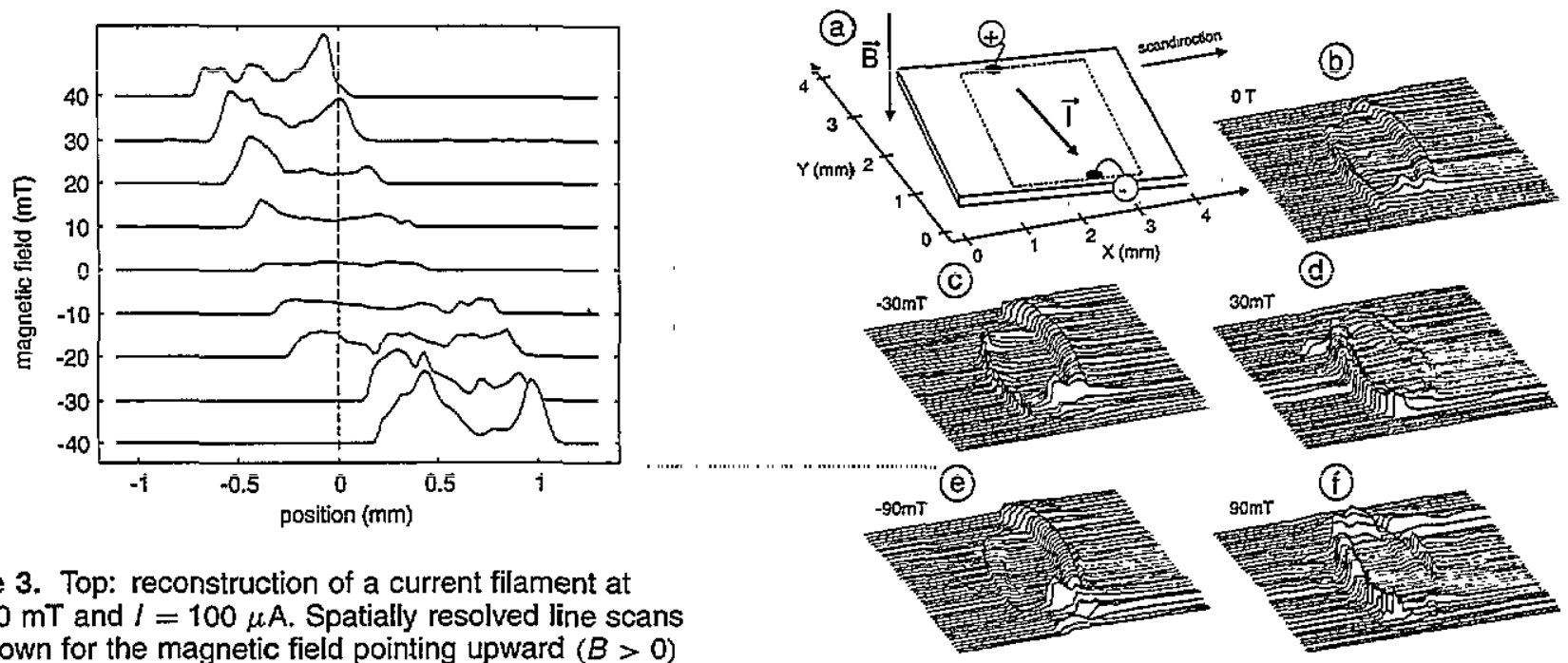

Figure 3. Top: reconstruction of a current filament at $B=40 \mathrm{mT}$ and $I=100 \mu \mathrm{A}$. Spatially resolved line scans are shown for the magnetic field pointing upward $(B>0)$ añu downinwarud $(B<0)$ andu for positive and negative currents. The double arrow in the centre indicates the symmetry of the filament if both current and magnetic field are inverted. Bottom: photosignal along a single line in the centre of the filament at $I=100 \mu \mathrm{A}$ for various positive (pointing upward) and negative (pointing downward) magnetic fields.

current-voltage characteristic field shown in figure 1. The recordings demonstrate again the symmetry of the filament displacement with respect to inversion of the orientation of the magnetic field.

Increasing the magnetic field strength up to about $100 \mathrm{mT}$ at a constant current of $100 \mu \mathrm{A}$ destabilizes the filament, yielding relaxation oscillations that are strictly periodic. The spatially well located structures in the photoresponse disappear.

Keeping the magnetic field strength constant and increasing the current causes a stiffening of the filament. In figure 4 the displacement of the centre of the filament is shown as function of the current at $B=40 \mathrm{mT}$. The displacement decreases with rising current and slowly approaches zero at about $0.8 \mathrm{~mA}$. Figure 5 displays a reconstruction of the current filament for a large current of $1 \mathrm{~mA}$, which is outside the scale of current-voltage characteristics shown in figure 1. Recordings are shown for $B=0 \mathrm{mT}, 30 \mathrm{mT}$ and $90 \mathrm{mT}$ with the magnetic field pointing upward and downward. At zero magnetic field (figure $5(b)$ ) the almost symmetric signal ridges indicate the borders of the filament. In the magnetic

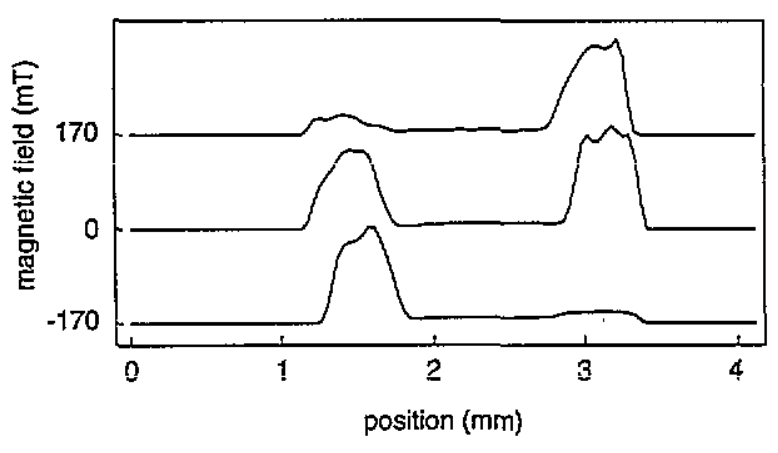

Figure 5. Top: reconstruction of a current filament at $l=1 \mathrm{~mA}$ for various magnetic fields. The direction of the current and the orientation of the positive magnetic field are indicated in (a), which shows the sample geometry. Bottom: photosignal along a single line in the centre of the filament for various positive (pointing upward) and negative (pointing downward) magnetic fields.

field the symmetry of the ridge-like structures is broken. With increasing magnetic field strength the response of the ridge on the side of the filament, which points in the direction of the Lorentz force, decays whereas the other ridge grows and gets wider. Inverting the magnetic field mirrors the spatial pattern at the axis of symmetry of the $B=0$ structure, as expected from a reverse of the Lorentz force. In contrast to the situation at small currents, the magnetic field does not laterally displace 
the filament. This is clearly observed in the recordings shown in the bottom diagram of figure 5 . Here the signal is plotted along a single line in the filament centre for $B=0$ and $\pm 170 \mathrm{mT}$. The magnetic field affects only the strength of the signal in the filament borders, and does not change at all the spatial distribution of the current flow as a whole.

\section{Discussion}

At zero magnetic field regular oscillations of the voltage across the sample are caused by oscillations of the electric field around the critical value for breakdown. This causes a repetitive ignition and extinction of current flow sharply localized in space along the shortest distance between the contacts. These oscillations may properly be described by a breathing filament [19]. At a sufficiently large average current a stable filament is formed when the impact ionization rate of shallow impurities is high enough to sustain a steady-state free carrier concentration far above the thermal equilibrium density.

The present experimental results reveal a mechanism by which such a filament is destabilized by a magnetic field oriented normal to the plane of the epitaxial layer. At small currents, slightly above the zero-field oscillatory regime in the current-voltage characteristic, the Lorentz force laterally displaces and stretches the filament. This distortion of the filament resembles that of a string being fixed at the two point contacts and subjected to a constant force field. We may attribute an internal tension $\sigma$ to a filament and equate the restoring force to the Lorentz force. For small elongations this yields $\sigma=\frac{1}{8}(j B / x) L^{2}$ where $j$ is the current density in the filament, $x$ the amplitude of the filament centre and $L$ the length of the filament. Taking the $x$ from the measurements, we may determine $\sigma$ as a function of the current. Results for $B=40 \mathrm{mT}$ are plotted in figure 4 (right ordinate scale) and demonstrate the stiffening of a filament with rising current. The point contacts generate a dipole-like electric field distribution in the semiconductor film. With rising magnetic field the lateral displacement of the filament increases more and more until the driving electric field drops below the critical field and the filament is torn. This occurs in the centre of the filament where the electric field is smallest. Now the current through the sample decreases and the voltage across the contacts increases, forming a new filament along the shortest distance between the contacts. This process is periodically repeated, yielding regular oscillations, which are different in type from the breathing filament at zero magnetic field. The transverse movement of the filament is not accompanied by a lateral current as previously assumed [14]. Rather the displacement of the filament is like the superposition of two solitary waves of opposite phase. On the side of the filament pointing in the direction of the Lorentz force the free carrier population is shifted to the high-conducting state by impact ionization of shallow donors. On the other side, where the Lorentz force tends to remove the electrons, the high-conducting phase can no longer be sustained and the filament border is withdrawn. The lateral velocity is determined by the difference between the impact ionization and recombination rates at the filament boundaries, which do not cancel as long as the filament moves [20, 21].

The stiffening of the filament with increasing average current may be understood from the distribution of charges that generate the Hall electric field. At low currents, just when a filament is formed, the electron temperature and mobility are not much different inside and outside the filament. Then the Lorentz force accumulates charges predominantly at the borders of the sample. This causes a lateral electric potential with constant slope $\phi=-E_{\mathrm{H}} x$ due to the Hall field $E_{\mathrm{H}}=E B \mu$, where $\mu$ is the low field mobility and $E$ the longitudinal driving electric field. The free carrier distribution is not affected by the Hall field because the Poisson equation, $\mathrm{d}^{2} \phi / \mathrm{d} x^{2}=0$, remains unchanged. If $E$ is homogeneous, as in the case of laterally smooth stripe contacts, the filament may drift freely in the direction of the Lorentz force. Such transverse motions of filaments with constant velocity have been observed in p-Ge [14] and have been numerically simulated assuming the mobility to be spatially constant [20]. For the present situation of point contacts $E$ decreases in the transverse direction. Therefore only a finite lateral displacement and an eventual disription may occur for a filament bound to point contacts.

For high currents, of the order of $1 \mathrm{~mA}$, the mobility in the filament $\mu_{\mathrm{h}}$ is much larger than that of the lowconductivity external regions $\mu_{1}$. This increase of the mobility is caused by the high electron temperature in the filament $[12,17]$ and the shielding of ionized impurity scattering due to the large electron density. A lower limit of the mobility may roughly be estimated from the current, the width of the filament and by assuming that all shallow donors in the filament are ionized. For $I=1 \mathrm{~mA}$ we find $\mu_{\mathrm{h}} \simeq 4 \times 10^{4} \mathrm{~cm}^{2} \mathrm{~V}^{-1} \mathrm{~s}^{-1}$, which is about the same as the equilibrium mobility at $77 \mathrm{~K}$. The mobility slightly below the critical voltage of breakdown, which corresponds to $\mu_{\mathrm{l}}$, is about one order of magnitude less than $\mu_{\mathrm{h}}$. Therefore charges are now accumulated mainly at the filament borders. The surface charge of the filament $E B\left(\mu_{\mathrm{h}}-\mu_{1}\right) \simeq E B \mu_{\mathrm{h}}$ is practically equal to the total charge separated by the Hall effect. Thus the potential due to the Hall field drops completely across the filament, keeping the inhomogeneous electron distribution fixed in space.

\section{Summary}

In summary we have observed a lateral displacement and stretching of a current filament due to the Lorentz force in a rather low mobility n-GaAs epitaxial layer. The bias current was applied to the sample by ohmic point contacts, which produce a single filament fixed to the contacts. For currents slightly larger than are necessary 
to form a filament, the elongation of the filament continues with increasing magnetic field strength until the filament is disrupted and reformed along a shorter distance between the contacts. This causes self-sustained oscillations which are strictly periodic. With higher currents the filament gets stiffer and the elongation decreases and finally vanishes even for high magnetic fields. We emphasize that this scenario of magneticfield-induced autonomous oscillations is not generally observed in n-GaAs. With several other samples of lower compensation and higher mobility no lateral displacement of a filament could be observed. The gross inhomogeneous current flow is not affected by the magnetic field. Instead, fluctuations at the filament border are induced, leading to quasiperiodic, frequencylocked and chaotic oscillations [12,13]: This dissimilar behaviour of materials differing in mobility results most probably from the charge separation by the Hall effect. If charges are accumulated at the filament borders then there is no net force on the filament and a lateral displacement of the filament does not occur. The charges at the filament borders, however, change the local electric fields and thus alter the impact ionization probability. This causes a disparity between the two boundaries and the inside of a filament, generating complex oscillations.

\section{Acknowledgments}

The authors wish to thank E Schöll and V Novák for helpful discussions. Financial support by the Deutsche Forschungsgemeinschaft is gratefully acknowledged.

\section{References}

[1] Kostial H, Ihn T, Asche M, Hey R, Ploog K and Koch F 1993 Japan. J. Appl. Phys. 32491

[2] Mayer K M, Gross R, Parisi J, Peinke J and Huebener R P 1987 Solid State Commun. 6355

[3] Mayer K M, Parisi J and Huebener R P 1988 Z. Phys. B 71171

[4] Brandl A, Völker M and Prettl W 1989 Appl. Phys. Lett. 55238

[5] Aoki K, Kobayashi T and Yamamoto K 1982 J. Phys. Soc. Japan $\mathbf{5 1} 2373$

[6] Teitsworth S W, Westervelt R M and Haller E E 1983 Phys. Rev. Lett. 51825

[7] Held G A, Jeffries C and Haller E E 1984 Phys. Rev. Lett. 521037

[8] Peinke J, Mühlbacher A, Huebener R P and Parisi J 1985 Phys. Lett. 108A 407

[9] Seiler D G, Littler C L, Justice R J and Milonni P W 1985 Phys. Lett. 108A 462

[10] Brandl A, Geisel T and Prettl W 1987 Europhys. Lett. 3 401

[11] Spangler J, Brandl A and Prettl W 1989 Appl. Phys. A 48 143

[12] Brandl A, Kröninger W, Prettl W and Obermair G 1990 Phys. Rev. Lett. 64212

[13] Brandl A and Prettl W 1991 Phys. Rev. Lett. 663044

[14] Spangler J, Margull U and Prettl W 1992 Phys. Rev. B 45 12137

[15] Clauss W, Rau U, Peinke J, Parisi J, Kittel A, Bayerbach M and Huebener R P 1991 J. Appl. Phys. 70 232

[16] Hüpper G, Pyragas K and Schöll E 1994 Phys. Rev. to be published

[17] Brandl A, Völker M and Prettl W 1989 Solid State Commun. 72847.

[18] Golubev V G and Prettl W 1991 Solid State Commun. 79 1035

[19] Proshin S A, Golubev V G, Würf S, Spangler J, Schilz A and Prettl W 1993 Semicond. Sci. Technol. 81298

[20] Schöll E and Drasdo D 1990 Z. Phys. B 81183

[21] Schimansky-Geier L, Zülicke Ch and Schöll E 1991 Z. Phys. B 84433 